Original Article

\title{
Diagnostic Value of Urinary Heparin Binding Protein in Urinary Tract Infection in Children.
}

\section{Ahmed M El-Refaey ${ }^{1}$, Amira H. Hagar ${ }^{1}$, Nermin Y Abo El-kheir ${ }^{2}$, Mayada S. Zeid ${ }^{1}$.}

1- Department of Pediatrics, Mansoura University Children's hospital, Faculty of Medicine, Mansoura University, Egypt.

2- Department of Clinical Pathology, Faculty of medicine, Mansoura University, Egypt.

\begin{abstract}
Introduction

Urinary tract infection is one of the most common bacterial infection in infants and children, diagnosis can be missed or delayed as urine cultures are time consuming.
\end{abstract}

\section{Aim of the Study}

Our aim is to assess the sensitivity and specificity of urinary heparin binding protein (U-HBP) in diagnosis of Urinary tract infection.

\section{Methods}

Acute febrile children with core temperature $\geq 38$ for less than 1 week were enrolled, from 1 month to 3 years from January to May 2016. Urinary HBP levels were assessed by ELISA in the supernatant stored aliquots of centrifuged freshly voided urine. Data was analyzed using SPSS 21.

\section{Results}

We found that median levels of U-HBP, were significantly higher in positive focus group in comparison to negative with $\mathrm{p}$ value $<0.001$, and that there's a significant relationship between the U-HBP and urine culture (gold standard) with good positive and negative predictive value.

\section{Conclusion}

Urinary heparin binding protein can be used as reliable biomarker for the detection of UTI at level 650 picogram/ml in infants and children with sensitivity (72.7\%) and specificity (81.2\%).

\section{Keywords}

Urinary tract infection, Urinary Heparin binding protein, Febrile children, Specificity, Sensitivity.

\section{Running title}

Urinary Heparin Binding Protein in Urinary Tract Infection

\section{Corresponding Author Amira H.I. Ali Hagar}

Department of Pediatrics, Mansoura University Children's hospital, Faculty of Medicine,

Mansoura University, Egypt.

Address: Elthanawya Street, Mansoura, Dakahlyia, Egypt.

Mobile no.: +20-01015033778

Email address: dr.amira.2015@mans.edu.eg

ORCID ID: 0000-0002-9448-1428

geget: The Journal of the Egyptian Society of Pediatric Nephrology and Transplantation (ESPNT)

geget https://geget.journals.ekb.eg/

Published by ESPNT http://espnt.net/

Cohosted by Egyptian Knowledge Bank https://www.ekb.eg 


\section{Introduction}

Diagnosis of UTI is difficult and may be missed in up to $50 \%$ of children presenting to primary care [1]. Diagnosis in pre- or early school-aged children is difficult, because a high percentage are preverbal, there is no specific symptoms, and collection of uncontaminated urine samples is not easy, especially in primary care settings where the availability of time and private space for young children is challenging [2].

Current management, which includes imaging, prophylaxis and prolonged follow-up, has placed a heavy burden on National Health Society (NHS) primary and secondary care resources. It is costly, depends on limited evidence and is annoying for children and distressing for their parents. The aim is to achieve more consistent clinical practice, based on definite diagnosis and appropriate management [3]. Urinalysis alone is not enough for diagnosing UTI. Infants with unexplained fever or voiding symptoms may have positive urinary cultures even when abnormal findings are not evident on dipstick testing and completeurinalysis $[4,5]$.

Nitrite test is a rapid indirect test especially important in diagnosis of asymptomatic bacteriuria such as E Coli, Klebsiella and Proteus [6]. Drawbacks are: 4 hours of bladder incubation are required for bacteria to convert nitrate to nitrite, in addition prior antibiotic use and competitive effect on ascorbic acid make it common to have a nitrite negative urine test result that later shows a positive urine culture result, and also the presence of nonnitrite producing micro-organisms as Pseudomonas, enterococci and S Saprophyticus [7] .

Urine culture is the gold standard for diagnosing UTI [8]. Urine cultures specify colony count and identify bacteria, and antimicrobial susceptibility. The bladder incubation time is an important determinant of the magnitude of the colony count [9].

When pyuria is present, $5 \times 10^{4} \mathrm{CFU}$ per $\mathrm{ml}$ of a single microorganism is significant in catheterized specimens but infection may be present with counts from $10 \times 10^{3}$ to $50 \times$ $10^{3} \mathrm{CFU} / \mathrm{mL}$. Although urine cultures are essential for all children, and adults with recurrent UTIs, treatment failures, or complicated UTIs, they may not be necessary as part of the evaluation of outpatients with uncomplicated UTIs [10]

Delayed or inefficient management of UTI may be associated with long-term complications such as renal scarring and renal insufficiency that could be prevented by early accurate diagnosis and prompt treatment. However, clinical manifestations of UTI in young children can be very subtle and nonspecific and diagnosing the condition can be challenging [2].

$\mathrm{HBP}$ is a protein of $37 \mathrm{kDa}$ stored in secretory and azurophilic granules of human neutrophils. When released from activated neutrophils, this multifunctional inflammatory mediator induces vascular leakage and acts as (1) a chemoattractant and (2) activator of monocytes. In addition, HBP has a broad antimicrobial activity and may also contribute to bacterial clearance by direct opsonization [11] .

Heparin-binding protein has been evaluated in clinical trials as a biomarker for different bacterial infections. Increased values of HBP in plasma, cerebrospinal fluid, and skin biopsies have been associated with severe sepsis, bacterial meningitis, and streptococcal skin infection [12].

Higher levels of urinary-HBP were noticed in patients with higher concentrations of bacteria in urine compared to patients with lower concentrations. This finding is in line with previous studies showing the direct effect of bacterial structures on the release of HBP [13]. U-HBP had also been used distinguish asymptomatic bacteriuria from symptomatic urinary tract infection (UTI), which leads to unnecessary antibiotic treatment [14].

\section{Methods}

This cross-sectional study was carried out in our center. It was conducted from January to May 2016. It comprised eighty one children, (52 were boys) and (29 were girls), thirty-seven cases had evident focus for bacterial infection by history and examination and enrolled as (-positive focus), forty four case were free from any evidence of bacterial infection by history and examination enrolled as (-negative focus).

Patients within the age group (3- 36 months), with fever $\geq 38^{\circ} \mathrm{C}$ with (positive focus group) or without (negative focus) clinically identifiable source of infection were included. Children with underlying immune deficiency, congenital anomalies or receiving immunosuppressive or prior antibiotic therapy will be excluded

Ethics approval and consent to participate: The study was permitted by the local ethical committee and under the Helsinki declaration of Bioethics and its later amendments. Informed consent (written form) was obtained from all participants' caregivers.

All patients were subjected to thorough history complete sepsis work up including CBC, CRP, urine analysis, urine culture, blood culture, and U-HBP was done by commercially available INOVA ELISA kits.

Urine was collected from catheterized patients. The catheter was clamped off to allow collection of freshly voided urine samples that were delivered immediately to microbiology unit and processed quickly. Macroscopic examination was done to detect color and aspect and direct microscopic examination of fresh film from the sediment was examined to detect pus cells, RBCs, epithelial cells urine strips to detect leukocyte esterase, nitrite, urobilinogen, protein, $\mathrm{PH}$, specific gravity.

Culture Urine samples were inoculated on to cysteine lysine electrolyte deficient (CLED) medium using a standard $0.01 \mathrm{ml}$ calibrated bacteriological loop. After 2448 hours of incubation at $37^{\circ} \mathrm{C}$, the number of colonies was estimated. A count of 103 or more $\mathrm{CFU} / \mathrm{ml}$ was considered significant.

Human heparin binding protein was done by commercially available INOVA ELISA kits (human azurocidin/heparin binding protein purchased from bioneovan Co., Ltd (daxing industry zone Beijing, china). Urine samples were collected, and preserved at $-20^{\circ} \mathrm{C}$, samples then were diluted to original density standard Diluted samples were added to testing wells, , samples were incubated for $30 \mathrm{mins}$ at $37^{\circ} \mathrm{C}$ then dried by swing, washing buffer was added this was repeated for 5 times. Chromogen solution A 50 $\mu$ l and Chromogen solution B $50 \mu \mathrm{l}$ were added to each well after 10 minutes stop solution 
was added, absorbance at $450 \mathrm{~nm}$ and within $15 \mathrm{~min}$ of adding stop solution was read. Reference range is 300-1125 $\mathrm{pg} / \mathrm{ml}$.

\section{Statistical Analysis}

Data were analyzed with SPSS version 21. Qualitative data were described using number and percent. Association between categorical variables was tested using Chi-square test.

Continuous variables were presented as mean \pm SD for parametric data and Median (Min-Max) for non-parametric data. The Comparison between more than two means was tested by ANOVA test while comparison between more than two medians was tested by Kruskal-Wallis test. Sensitivity and specificity at different cut-off point were tested by Roc curve, the statistical significance detected when the probability of error is less than $5 \%(\mathrm{p}<0.05)$.

\section{Results}

This study comprised eighty-one cases of febrile infants and children of matched age and sex with or without focus for infection, including 37 infants and children who had evident focus for infection (positive focus) with median age 1year and 6 months. 44 infants and children who were free from any evidence of infection (negative focus) with median age 2 years.

The observed focuses from both symptomatology and clinical examination comprise 14 cases of acute tonsillitis, 7 cases of acute gastroenteritis, 4 cases of acute otitis media, 5 cases of pneumonia, 3 cases of acute bronchiolitis, and 4 cases of UTI presented with dysuria.

This study shows that both groups were sex and age matched $(\mathrm{P}=0.110, \mathrm{P}=0.191)$ respectively as shown in (Table 1), there's statistically significant difference in results of WBCS, CRP and urine pus cells between both groups as in (Table 2) and no significant difference between the two groups $(\mathrm{P}=0.051, \mathrm{P}=0.36)$ as regard results of both urine and blood culture respectively as shown in (Table 3 ).

The most common pathogen in urine cultures was E. coli $(53.3 \%),(43.7 \%)$ in both group I and II respectively, non-E.coli represent $(46 \%),(56.2 \%)$ in both group I and II respectively.Non-E.coli pathogen include: Serratia, Klebsiella, micrococci, Citrobacter, and pseudomonas as shown in (Table 4).

The most common pathogens in blood culture were E.coli $75 \%$ and pneumococci $25 \%$ in group I and also E.coli $71.4 \%$ in group II together with pneumococci $14.3 \%$ and Serratia $14.3 \%$. The current study results show that there's no significant correlation between U-HBP and WBCs, CRP but there's a statistically significant correlation between $\quad$ U-HBP \& urine pus cells ( $p$ value $=0.01$ ) as shown in (Table 5).

The study shows that there's significant relationship between results of urine cultures and results of U-HBP with $\mathrm{p}$ value $<0.001$ as shown in (Table 6). The study shows the diagnostic accuracy of HBP for detection for UTI in febrile children aged 3-36 months with sensitivity (72.2\%), specificity $(81.2 \%)$ at HBP level 650picogram $/ \mathrm{ml}$, area under curve was 0.648 (95\% CI $0.52-0.77)$ as shown in (Figure 1).

Table 1 Comparison between studied group regarding demographic data

\begin{tabular}{|c|c|c|c|c|c|c|}
\hline \multirow{2}{*}{ Variables } & \multicolumn{2}{|c|}{ Positive focus $(n=37)$} & \multicolumn{2}{|c|}{ Negative focus $(n=44)$} & \multirow{2}{*}{ Test of sig. } & \multirow{2}{*}{ p-value } \\
\hline & No & $\%$ & No & $\%$ & & \\
\hline Male & 27 & 73.0 & 25 & 56.8 & \multirow{2}{*}{$\chi^{2}=4.41$} & \multirow{2}{*}{0.110} \\
\hline Female & 10 & 27.0 & 19 & 43.2 & & \\
\hline Median & \multicolumn{2}{|c|}{1.6} & \multicolumn{2}{|c|}{2} & \multirow{2}{*}{$\mathrm{KW}=3.316$} & \multirow{2}{*}{0.191} \\
\hline Min-Max & \multicolumn{2}{|c|}{$0.3-3$} & \multicolumn{2}{|c|}{$0.5-13$} & & \\
\hline
\end{tabular}

$\chi^{2}$ : Chi square test, KW: Kruskal-Wallis test

Table 2 Comparison between studied group regarding WBCs, CRP, pus cells in urine

\begin{tabular}{|c|c|c|c|c|}
\hline Variables & Positive focus $(n=37)$ & Negative focus $(n=44)$ & Test of sig. & p-value \\
\hline \multicolumn{5}{|c|}{ WBCs } \\
\hline Median & 10 & 8.95 & \multirow{2}{*}{$\mathrm{KW}=11.75$} & \multirow{2}{*}{ 0.003* } \\
\hline Min-Max & $3.5-40$ & $1.8-30$ & & \\
\hline \multicolumn{5}{|c|}{ CRP } \\
\hline Median & 25 & 0 & \multirow{2}{*}{12.053} & \multirow{2}{*}{$0.002 *$} \\
\hline Min-Max & $0-112$ & $0-160$ & & \\
\hline \multicolumn{5}{|c|}{ Pus cells in urine } \\
\hline Median & 20 & 18 & \multirow{2}{*}{9.546} & \multirow{2}{*}{$0.016 *$} \\
\hline Min-Max & $0-100$ & $0-25$ & & \\
\hline
\end{tabular}


Table 3 Comparison between positive and negative focus groups as regard isolated of urine and blood culture

\begin{tabular}{|c|c|c|c|c|c|c|}
\hline & \multicolumn{2}{|c|}{$\begin{array}{c}\text { Positive focus } \\
(\mathbf{n}=\mathbf{3 7})\end{array}$} & \multicolumn{2}{|c|}{$\begin{array}{l}\text { Negative focus } \\
(n=44)\end{array}$} & \multirow[t]{2}{*}{$\chi^{2}$} & \multirow[t]{2}{*}{ p-value } \\
\hline & No & $\%$ & No & $\%$ & & \\
\hline \multicolumn{7}{|c|}{ Urine culture } \\
\hline Positive & 15 & 40.5 & 16 & 36.4 & \multirow{2}{*}{5.961} & \multirow{2}{*}{0.051} \\
\hline Negative & 22 & 59.5 & 28 & 63.6 & & \\
\hline \multicolumn{7}{|c|}{ Blood culture } \\
\hline Positive & 4 & 10.8 & 7 & 15.9 & \multirow{2}{*}{2.036} & \multirow{2}{*}{0.361} \\
\hline Negative & 33 & 89.2 & 37 & 84.1 & & \\
\hline
\end{tabular}

Table 4 Comparison between positive \& negative focus groups as regard results microorganism from urine and blood cultures

\begin{tabular}{|c|c|c|c|c|c|c|}
\hline \multirow[t]{2}{*}{ Variables } & \multicolumn{2}{|c|}{$\begin{array}{c}\text { Positive focus } \\
(\mathbf{n}=\mathbf{3 7})\end{array}$} & \multicolumn{2}{|c|}{$\begin{array}{c}\text { Negative focus } \\
(n=44)\end{array}$} & \multirow[t]{2}{*}{$\chi^{2}$} & \multirow[t]{2}{*}{ p-value } \\
\hline & No & $\%$ & No & $\%$ & & \\
\hline \multicolumn{5}{|l|}{ Urine culture } & \multirow{3}{*}{12.036} & \multirow{3}{*}{0.303} \\
\hline E coli & 8 & 53.3 & 7 & 43.7 & & \\
\hline Non E.coli & 7 & $46 \%$ & 9 & 56.2 & & \\
\hline \multicolumn{7}{|l|}{ Blood culture } \\
\hline S.pneumonia & 3 & 75 & 5 & 71.4 & \multirow{2}{*}{2.81} & \multirow{2}{*}{0.833} \\
\hline H.influenza & 1 & 25 & 2 & 28.6 & & \\
\hline \multicolumn{7}{|c|}{ Organism in urine culture } \\
\hline Serratia & 0 & 0.0 & 2 & 12.5 & \multirow{5}{*}{10.046} & \multirow{5}{*}{0.623} \\
\hline Klebsiella & 4 & 26.6 & 6 & 37.5 & & \\
\hline Micrococci & 1 & 6.7 & 0 & 0.0 & & \\
\hline Citrobacter & 1 & 6.7 & 1 & 6.3 & & \\
\hline Pseudomonas & 1 & 6.7 & 0 & 0.0 & & \\
\hline
\end{tabular}

Table 5 Correlation between HBP and WBCs, CRP, UA

\begin{tabular}{|c|c|c|}
\hline & \multicolumn{2}{|c|}{ U-HBP } \\
\cline { 2 - 3 } & $\mathbf{R}$ & $\mathbf{P}$ \\
\hline WBCs & 0.134 & 0.204 \\
\hline CRP & 0.092 & 0.387 \\
\hline UA/pus cells & 0.812 & $\mathbf{0 . 0 1} *$ \\
\hline
\end{tabular}

Table 6 Comparison between positive and negative U-HBP as regard results of urine culture

\begin{tabular}{|c|c|c|c|c|c|c|}
\hline & \multicolumn{2}{|c|}{$\begin{array}{c}\text { Positive urine culture } \\
(\text { no }=33)\end{array}$} & \multicolumn{2}{|c|}{$\begin{array}{l}\text { Negative urine culture } \\
(\text { no }=48)\end{array}$} & \multirow{2}{*}{$\chi^{2}$} & \multirow{2}{*}{ p-value } \\
\hline & No & $\%$ & No & $\%$ & & \\
\hline \multicolumn{7}{|l|}{ U-HBP } \\
\hline Positive & 32 & $96.9 \%$ & 13 & $27 \%$ & \multirow{2}{*}{18.897} & \multirow{2}{*}{$0.001 *$} \\
\hline Negative & 1 & $3.1 \%$ & 35 & $73 \%$ & & \\
\hline
\end{tabular}

$$
\chi^{2}: \text { Chi square test }
$$

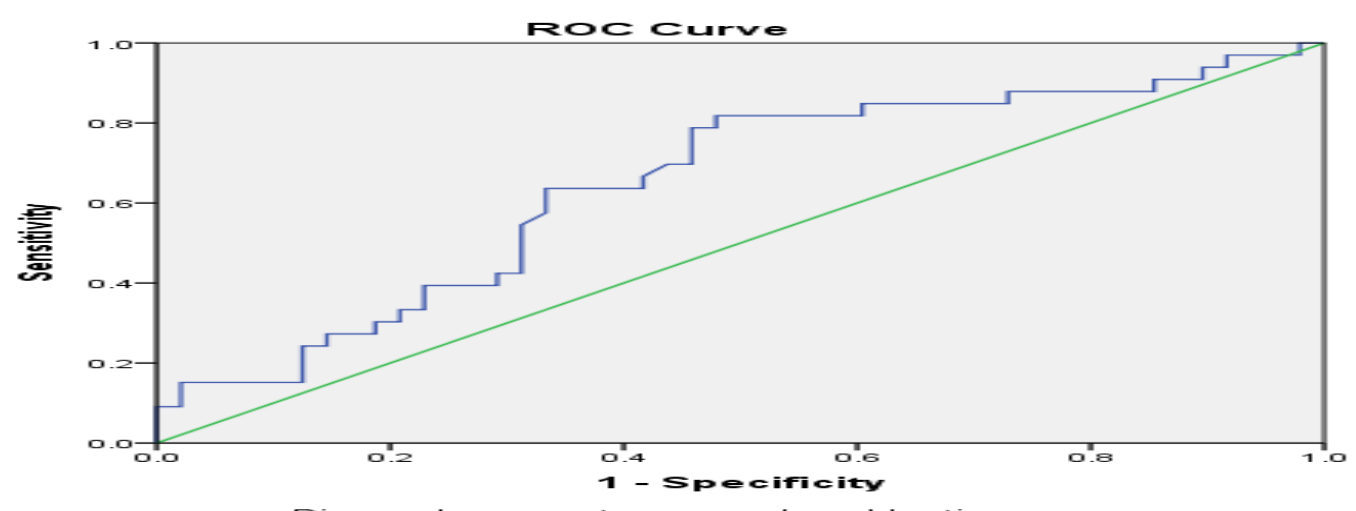

Diagonal segments are produced by ties

Figure 1 ROC curve for prediction of urine culture-positive UTI by U-HBP 


\section{Discussion}

When febrile infant and young children below 3 years are presented to the clinician, they are usually faced with the dilemma of deciding whether a workup is necessary and if antibiotic therapy is indicated. The most helpful screening test would predict which children are at a higher risk of bacterial infection and consequently, in need for further workup and possible antibiotics [15].

UTI remains the most common occult bacterial infection especially in this age group who are almost pre- or nonverbal and urinary symptoms are almost absent. Although methods of screening of children for UTI is well known, it is also time consuming. Furthermore, there are lots of opinions about the best testing policy for diagnosing UTI. Moreover, the results of the urine culture are delayed by 24 to 48 hours. Which raise the importance of a rapid dependable diagnostic test $[16,17]$.

This study showed that urinary tract infection (UTI) was a common cause of fever with prevalence rate $40.5 \%$ in positive focus group and $36.4 \%$ in negative focus group. In agreement with these finding, UTI accounted for $80 \%$ of infant and children of age group 3 months to 3 years presenting with fever followed by pneumonia and bacteremia [18]. UTI was the most common in infants below 3 months followed by bacteremia and meningitis also in Gomez et al [19]. There was no statistically significant difference found among organisms isolated from positive urine culture. These organisms included Escherichia coli $53.3 \%$ and non E.coli $46 \%$ which were $26.6 \%$ for Klebsiella, $6.7 \%$ for micrococci, citrobacter and pseudomonas, this correlates with other studies. Bryan et al reported $\mathrm{E}$. coli as the common urinary pathogen in $85 \%$ of cases [20].

The relatively lower percentage of E. coli in urine culture in our study compared to other previously mentioned studies can be explained by the relative male predominance $(64 \%)$ of the study group, where female gender with short urethra facilitate mobilization and travel of fecal E.coli from GIT to the urinary tract, other studies show slightly female predominance [21].

As regard blood cultures, this study showed that $10.8 \%$ of positive focus group had positive blood cultures and $15.9 \%$ of negative focus group had positive blood cultures with no statistically significant difference between both groups.

The most common organism was strept pneumoniae followed by $\mathrm{H}$ influenza, this finding is consistent with Arora et al who found that positive blood culture constitute $11.6 \%$ in a study group formed of 150 infants and children aged from 2 month to 3 years presented with fever without focus with Streptococcus pneumoniae accounting for most cases followed by Salmonella species and Neisseria meningitides [22] .

Levels of U-HBP were significantly higher among positive focus group in comparison to the negative focus group, which can be explained by the fact that fever in negative focus group can be caused by viral causes where there's no role for U-HBP which is secreted from neutrophil this is consistent with Kjölvmark et al who found that UHBP level was significantly higher in patient with febrile UTI in comparison to non UTI group [23].

It was also found that there's no significant correlation between U-HBP and WBCs, CRP. In contrast to finding of Kjölvmark et al Lertdumrongluk et al who found that levels of U-HBP were significantly correlated with WBCs and ANC [23, 24].

There's a statistically significant correlation between UHBP and urine pus cells is consistent with finding of Kjölvmark et and al Lertdumrongluk et al who found that U-HBP levels were positively related with the degree of UWBC. This result was expected because U-HBP is secreted from neutrophils and also due to increased vascular permeability caused by U-HBP, which Can augment the trans endothelial migration of neutrophils [14].

It is vague whether U-HBP is secreted locally within the urinary tract or is the cleared by the kidney as a result of increased serum U-HBP, possibility that U-HBP might be secreted locally by neutrophils activated within the urinary tract is supported by these findings, and this is also supported by the finding that U-HBP levels were higher in patients with UTI than in patients with other infections but absence of data on serum HBP so we didn't reach a firm assumption on the source of U-HBP.

\section{Conclusion}

In conclusion to this study, it was found that Urinary heparin binding protein can be used as a reliable biomarker for diagnosis of UTI at level 650 picogram $/ \mathrm{ml}$ in infants and children with sensitivity (72.2\%) and specificity (81.2\%).

\section{Recommendation}

We recommend screening for urinary tract infection in acute febrile children aged 1 month to 3 years using U-HBP and starting antibiotic according to the local sensitivity (if positive) till results of urine culture is revealed.

Abbreviations
\begin{tabular}{|c|c|}
\hline ANC & Absolute neutrophilic count \\
\hline CRP & C reactive protein \\
\hline CFU & Colony forming unit \\
\hline U-HBP & Urinary heparin binding protein \\
\hline UTI & Urinary tract infection \\
\hline
\end{tabular}




\section{References}

1. Coulthard, M.G., et al. A nurse led education and direct access service for the management of urinary tract infections in children: prospective controlled trial. BM M. Lakhanpaul, and K. Verrier-Jone J, 2003. 327(7416): p. 656-0.

2. Mori, R., s, Diagnosis and management of urinary tract infection in children: summary of NICE guidance. BMJ, 2007. 335(7616): p. 395-397.

3. Bowpitt,G.,etal.,Supporting Children and Families: Lessons from Sure Start for Evidence-based Practice in Health, SocialCare and Education. Jessica Kingsley Publishers, 2007. 15.

4. Anad, F.Y., A simple method for selecting urine samples that need culturing. Annals of saudi medicine, 2001. 21(1-2): p. 104-105.

5. Bachur, R. and M.B. Harper, Reliability of the urinalysis for predicting urinary tract infections in young febrile children. Archives of Pediatrics Adolesc Med, 2001. 155(1):60-65.

6. Doern, C.D. and S.E. Richardson, Diagnosis of Urinary Tract Infections in Children. J Clin Microbiol, 2016. 54(9): p. 2233 - .2242

7. Zorc, J.J., D.A. Kiddoo, and K.N. Shaw, Diagnosis and management of pediatric urinary tract infections. J Clin Microbiol Rev, 2005. 18(2): p. 417-422.

8. Roberts, K.B., Urinary tract infection: Urinary Tract Infection: Clinical Practice Guideline for the Diagnosis and Management of the Initial UTI in Febrile Infants and Children 2 to 24 Months Pediatrics, 2011. 128(3): p. 595-610.

9. Foxman, B., Epidemiology of urinary tract infections: incidence, morbidity, and economic costs. American Journal Med :(1)113.2002 ‘p. 5-13.

10. Tapper, H., Secretion of heparin-binding protein from human neutrophils is determined by its localization in azurophilic granules and secretory vesicles. Blood, 2002. 99(5): p. 1785-1793.

11. Linder, A., et al., Elevated plasma levels of heparinbinding protein in intensive care unit patients with severe sepsis and septic shock. Crit Care, 2012; 16(3):R90. doi: 10.1186/cc11353

12. Herwald, H., et al., M protein, a classical bacterial virulence determinant, forms complexes with fibrinogen that induce vascular leakage. Cell, 2004. 116(3): p. 367-379.

13. Kjölvmark, C., et al., Distinguishing asymptomatic bacteriuria from urinary tract infection in the elderlythe use of urine levels of heparin-binding protein and interleukin-6. Diagnostic microbiology and infectious disease, 2016. 85(2): p. 243-248.

14. Hoberman, A., et al., Prevalence of urinary tract infection in febrile infants. J Pediatr, 1993. 123(1): p. 17-23.

15. Hoberman, A., et al., Oral Versus Initial Intravenous Therapy for Urinary Tract Infections in Young Febrile Children. Pediatrics, 1999. 104(1): p. 79-86.

16. Gorelick, M.H. and K.N. Shaw, Screening tests for urinary tract infection in children: a meta- analysis. Pediatrics, 1999. 104(5): p. e54-e54.

17. Machado ‘B.M., et al., Fever without source: evaluation of a guideline. Jornal de Pediatria, 2009. 85(5): p. 426432.

18. Gómez, B., et al., Blood Culture and Bacteremia Predictors in Infants Less Than Three Months of Age With Fever Without Source. The Pediatric Infectious Disease Journal, 2010. 29(1): p. 43-47.

19. Moreno, E., et al., Relationship between Escherichia coli strains causing urinary tract infection in women and the dominant faecal flora of the same hosts. Epidemiol Infect, 2006. 134(5): p. 1015-1023.

20. Moreno, E., et al., Relationship between Escherichia coli strains causing urinary tract infection in women and the dominant faecal flora of the same hosts. Epidemiol Infect, 2006. 134(5): p. 1015-1023.

21. Arora, R. and P. Mahajan, Evaluation of child with fever without source: review of literature and update. Pediatr Clin North Am, 2013. 60(5): p. 1049-1062.

22. Kjölvmark, C., P. Åkesson, and A. Linder, Elevated urine levels of heparin-binding protein in children with urinary tract infection. Pediatric Nephrology, 2012. 27(8): p. 1301-1308

23. Lertdumrongluk, K., et al., Diagnostic accuracy of urine heparin binding protein for pediatric acute pyelonephritis. Eur J Pediatr, 2015. 174(1): p. 43-48 


\section{Statements}

Ethics approval and consent to participate:

This study protocol and the consents were approved and deemed sufficient by the Ethical Committee of Mansoura university children's hospital and informed written consent was obtained in every case from their legal guardians.

\section{Consent for publication}

"Not applicable"

\section{Availability of data and material}

"Not applicable"

\section{Conflict of interest}

The authors declare no conflict of interest

\section{Funding}

The authors declare that this research work did not receive any fund.

\section{Acknowledgement}

Authors would like to thank all patients and their family members for their valuable contributions to the study.

$\begin{array}{lc}\text { Submitted } & 18 / 1 / 2020 \\ \text { Revised } & 7 / 3 / 2020 \\ \text { Accepted } & 26 / 3 / 2020 \\ \text { Published online } & 30 / 6 / 2020\end{array}$

